\title{
Fallo intestinal en el paciente crítico: reconocimiento, abordaje multidisciplinario y unidades dedicadas
}

Intestinal failure in critical patients: recognition, multidisciplinary approach and dedicated units

Insuficiência intestinal no paciente crítico: reconhecimento, abordagem

multidisciplinar e unidades dedicadas

Aldo Agüero ${ }^{1}$, Kenia Infante ${ }^{2}$

\section{Resumen}

Introducción: en el paciente crítico el intestino es protagonista de la respuesta al estrés. Los síntomas de insuficiencia intestinal en las unidades de cuidados intensivos no son específicos y no se incluyen en escalas de gravedad comúnmente usadas, sin embargo, el 62\% de los pacientes críticos presenta síntomas gastrointestinales al menos una vez al día. Demostrándose la relación existente entre la aparición de problemas gastrointestinales y la mortalidad en pacientes críticos.

Objetivo: proporcionar el conocimiento teórico necesario sobre el fallo intestinal como entidad exclusiva, su reconocimiento en el paciente grave, clasificación y enfoque multidisciplinario del tratamiento.

Métodos: se realizó una revisión cualitativa y sistemática en los idiomas inglés y español de la literatura publicada y actualizada hasta febrero del 2020.

Conclusiones: las alteraciones gastrointestinales son frecuentes en el paciente crítico y un determinante en su mortalidad. Reconocer el fallo y disfunción intestinal permite optimizar el tratamiento, reducir la mortalidad e incidencia de complicaciones, las unidades dedicadas con un equipo de experiencia en el manejo del fallo intestinal agudo constituyen un aspecto clave en este sentido.

Palabras clave: Fallo intestinal

Disfunción intestinal

Enfermedades intestinales

Unidades de cuidados intensivos
Key words: Intestinal failure

Bowel dysfunction

Intestinal diseases

Intensive Care Units

1. Servicio de Terapia Intensiva. Hospital Clínico Quirúrgico "Hermanos Ameijeiras". La Habana, Cuba.

2. Servicio de Pediatría. Policlínico Universitario Boyeros. La Habana, Cuba.

Correspondencia: Aldo Agüero. Correo electrónico: aldoagueromilanes1979@gmail.com

Los autores declaran no tener conflicto de intereses.

No existió financiación.

Recibido: $7 / 12 / 202$

Aprobado 24/4/2021

Attribution-NonCommercial 4.0 International (CC BY-NC 4.0) 


\section{Introducción}

El sistema gastrointestinal (GI), además de la absorción, digestión de nutrientes y secreción de gran cantidad de hormonas, es el mayor compartimiento mucoso del sistema inmune y una barrera al paso de antígenos, tóxicos, microorganismos y sus toxinas desde la luz intestinal a la circulación sistémica ${ }^{(1)}$. Esta "barrera intestinal" constituye un sistema defensivo compuesto por elementos extracelulares ( $\mathrm{pH}$ intraluminal; secreciones gástricas, pancreáticas y biliares; peristaltismo; capa de moco, agua y glicocálix que previene la adhesión e invasión por microorganismos y contiene inmunoglobulina A secretora [IgAs]) y celulares (epitelio intestinal, tejido linfoide asociado al intestino, microbiota intestinal y el sistema nervioso entérico organizado en una red interconectada de neuronas y células gliales) ${ }^{(2)}$.

La microbiota intestinal es un ecosistema diverso que comprende bacterias, arqueas, hongos, virus y bacteriófagos, entre sus funciones se reconocen: metabólicas, tróficas y de protección ${ }^{(3)}$. El microbioma se refiere al número total de microorganismos, su material genético y las condiciones ambientales circundantes, mientras que la colección de genes y genomas de una microbiota constituye el metagenoma ${ }^{(4)}$.

En el paciente crítico el intestino es protagonista de la respuesta al estrés y todos los elementos de la barrera intestinal son afectados, condicionando alteraciones sistémicas, disminución de la inmunidad, situación proinflamatoria, susceptibilidad a infecciones, sepsis y síndrome de fracaso multiorgánico ${ }^{(5)}$. Los síntomas de insuficiencia intestinal en las unidades de cuidados intensivos (UCI) no son específicos y no se incluyen en escalas de gravedad comúnmente usadas, como el Sequential Organ Failure Assessment (SOFA), sin embargo, el $62 \%$ de los pacientes críticos presenta síntomas GI al menos una vez al día ${ }^{(6)}$. Demostrándose la relación existente entre la aparición de problemas GI y la mortalidad en pacientes críticos $(43,7 \%$ frente a $5,3 \%$ de los pacientes sin alteraciones $\mathrm{GI})^{(7)}$.

Fleming y Remington, en 1981, usaron por primera vez el término "fallo intestinal". Hasta el 2014 habían menos de 1.000 referencias al tema, recientemente la Sociedad Europea de Nutrición Clínica y Metabolismo (ESPEN) ofrece una nueva definición y clasificación del fallo intestinal $(\mathrm{FI})^{(8)}$.

\section{Metodología}

El objetivo de esta revisión es proporcionar el conocimiento teórico necesario sobre el fallo intestinal como entidad exclusiva, su reconocimiento en el paciente grave, su clasificación y el enfoque multidisciplinario del tratamiento.
Se realizó una revisión cualitativa y sistemática en inglés y español de las bases de datos PubMed/MEDLINE, EMBASE, Scopus y Cochrane Central para identificar la documentación publicada sobre el tema, como artículos de revisión, ensayos clínicos, guías clínicas y estudios observacionales entre el mes de octubre de 2000 hasta noviembre de 2020. Como criterios de búsqueda se incluyeron los siguientes descriptores: "fallo intestinal", "disfunción intestinal", "insuficiencia intestinal", "intestinal insufficiency", "intestinal failure", "intestinal dysfunction". Se revisaron los resúmenes y en casos necesarios los artículos completos, considerándose los que incluían recomendaciones sobre fallo, disfunción o insuficiencia intestinal.

\section{Resultados}

En total, se revisaron 97 citas y resúmenes para su inclusión (53 de PubMed/MEDLINE, 16 de EMBASE, 7 de Scopus y 21 de Cochrane Central). De estos fueron seleccionados cuatro guías clínicas, seis ensayos clínicos aleatorizados y 11 estudios observacionales controlados.

\section{Desarrollo y discusión}

Los pacientes graves con FI requieren una intervención multidisciplinaria y unidades dedicadas con experiencia. Este enfoque se asocia a menor incidencia de complicaciones y mortalidad ${ }^{(8,9)}$.

\section{Definición}

La ESPEN ${ }^{(8)}$ ha definido el FI como la reducción de la función intestinal bajo el mínimo necesario para la absorción de macronutrientes y/o agua y electrolitos, requiriéndose suplementación intravenosa (IV) para mantener la salud y/o el crecimiento. La reducción de la función intestinal de absorción que no precisa suplementación IV, puede considerarse como disfunción intestinal.

\section{Clasificación y etiología}

Según la duración del soporte nutricional y/o de agua y electrolitos IV, algunos pacientes con FI necesitarán soporte por un corto período de tiempo mientras se recupera la función intestinal, como sucede en algunos postoperatorios de cirugía abdominal o con otras patologías (neumonía, traumatismo craneoencefálico, pancreatitis aguda). En otros pacientes más complejos y metabólicamente inestables, el soporte IV puede extenderse por un período mayor de tiempo y requerirán atención multidisciplinaria, generalmente en UCI. Un grupo menor de casos, estables metabólicamente, podrán requerir soporte nutricional IV durante meses o incluso años. 
Tabla 1. Clasificación funcional y etiología.

\begin{tabular}{|c|c|c|c|}
\hline Subtipo & Descripción & Etiología & Duración soporte IV \\
\hline FI agudo tipo I & $\begin{array}{l}\text { Condición aguda, autolimitada, } \\
\text { reversible al corregir las otras } \\
\text { disfunciones orgánicas o la causa que } \\
\text { lo origina, requiere soporte IV por un } \\
\text { corto periodo de tiempo. }\end{array}$ & $\begin{array}{l}\text { Íleo postoperatorio (10-30\%). Asociado } \\
\text { a enfermedades graves (trauma } \\
\text { craneoencefálico, neumonía, } \\
\text { pancreatitis, etcétera.) }\end{array}$ & Menor a 28 días \\
\hline Fl agudo tipo II & $\begin{array}{l}\text { Condición aguda prolongada, puede } \\
\text { ser contínuum del tipo I, pacientes } \\
\text { metabólicamente inestables, } \\
\text { usualmente en UCI, requieren } \\
\text { evaluación multidisciplinaria y soporte } \\
\text { nutricional IV durante semanas o } \\
\text { meses. }\end{array}$ & $\begin{array}{l}\text { Complicaciones quirúrgicas (32\%) } \\
\text { Enfermedad de Crohn (21\%) } \\
\text { Alteraciones de la motilidad (14\%) } \\
\text { Isquemia intestinal (13\%) } \\
\text { Enfermedades malignas }(8 \%) \\
\text { Enteritis por radiación }(2 \%) \\
\text { Enfermedad celíaca }(2 \%) \text {. } \\
\text { Otras (8\%) }\end{array}$ & Mayor a 28 días - Meses \\
\hline FI crónico o tipo III & $\begin{array}{l}\text { Pacientes estables metabólicamente, } \\
\text { requieren soporte intravenoso (nutrición } \\
\text { parenteral domiciliaria) durante meses } \\
0 \text { años. Puede ser reversible o } \\
\text { irreversible. }\end{array}$ & $\begin{array}{l}\text { Complicaciones quirúrgicas }(28,8 \%) \\
\text { Enfermedad inflamatoria intestinal } \\
(22,6 \%) \\
\text { Otras (enteritis por radiación, esclerosis } \\
\text { sistémica, linfangiectasia intestinal, } \\
\text { poliposis familiar, etc.) }\end{array}$ & $\begin{array}{l}\text { Meses - años. Usualmente: nutrición } \\
\text { parenteral domiciliaria. }\end{array}$ \\
\hline
\end{tabular}

Se identifican tres subtipos funcionales de FI (tabla 1), según el tiempo de duración y las características fisiopatológicas se reconocen cinco condiciones capaces de ocasionarlo (tabla 2).

Esta clasificación de la $\operatorname{ESPEN}^{(8)}$ fue resultado del trabajo de dos grupos de estudio: uno en 1992, “Grupo de falla intestinal crónica y nutrición artificial en el hogar", y otro en 2010, "Grupo de falla intestinal aguda".

En la tabla 1 se integra la etiología según el estudio británico de Lal y colaboradores ${ }^{(10)}$, estas enfermedades se resumen en cinco condiciones fisiopatológicas ${ }^{(8)}$ (tabla 2) y pueden coincidir más de una en un paciente con FI.

Según la severidad, el grupo de trabajo de problemas abdominales de la Sociedad Europea de Medicina Intensiva (European Society of Intensive Care Medicine, ESICM) propuso la clasificación de las alteraciones gastrointestinales agudas del paciente crítico en cuatro $\operatorname{grados}^{(11)}$ :

- Grado I (riesgo): náuseas posoperatorias o vómitos durante los primeros días poscirugía; ausencia de sonidos intestinales, disminución de motilidad intestinal en la fase precoz del shock.

- Grado II (disfunción GI): gastroparesia, aumento del residuo gástrico, parálisis del tracto GI, diarrea, aumento de la presión intraabdominal (PIA) entre 12-15 mmHg, sangrado digestivo. Existe intoleran- cia a la nutrición enteral (NE) (cuando a las 72 horas no se han podido aportar $20 \mathrm{kcal} / \mathrm{kg}$ ).

- Grado III (fallo intestinal agudo): persistencia de la intolerancia a pesar de intervención terapéutica, residuo gástrico aumentado, parálisis GI, distensión intestinal, elevación de la PIA (15-20 mmHg), y presión de perfusión abdominal(PPA) baja $(<60$ $\mathrm{mm} \mathrm{Hg}$ ).

- Grado IV (fallo intestinal agudo): isquemia intestinal con necrosis, hemorragia GI, shock hemorrágico, síndrome de Ogilvie, síndrome compartimental abdominal (SCA). Riesgo vital inmediato.

Esta clasificación considera parámetros objetivos como la PIA y la presencia de hipertensión abdominal o SCA en el diagnóstico de FIA. La hipertensión abdominal se diagnostica entre el 38\%-45\% de todos los pacientes adultos en $\mathrm{UCI}^{(12,13)}$ y la incidencia del SCA varía con la gravedad de la lesión; reportándose entre 10\%-35\% de los pacientes críticos sin lesión abdominal directa y en $30 \%$ después de una cirugía o traumatismo abdominal mayor con una mortalidad aproximada de $50 \%{ }^{(13)}$.

Ambas clasificaciones no son excluyentes, existiendo concordancia entre el tipo I de la clasificación funcional por la ESPEN ${ }^{(8)}$ y el grado I de la ESICM $^{(11)}$. Resulta complejo establecer una clasificación basada en la severidad del FI por la falta de marcadores de gravedad e in- 
Tabla 2. Clasificación fisiopatológica.

\begin{tabular}{ll}
\hline Condición fisiopatológica & \multicolumn{1}{c}{ Ejemplos } \\
\hline Intestino corto & $\begin{array}{l}\text { Resección quirúrgica extensa: Enfermedad de Crohn, infarto mesentérico, enteritis por } \\
\text { radiación, trauma abdominal, vólvulo, etc. } \\
\text { Congénito: atresia intestinal, onfalocele, gastrosquisis, malformación intestinal. }\end{array}$ \\
\hline Fístula intestinal & $\begin{array}{l}\text { latrogénicas, traumáticas, enfermedad de Crohn, neoplasias (primarias o metástasicas), } \\
\text { inflamatorias (enfermedad de Crohn, pancreatitis, enfermedad diverticular, etc.) }\end{array}$ \\
\hline Dismotilidad intestinal & $\begin{array}{l}\text { Aguda: posoperatorio de cirugía abdominal, respuesta inflamatoria sistémica, } \\
\text { enfermedades neurológicas. } \\
\text { Pseudobstrucción intestinal crónica (síntomas obstructivos de al menos } 6 \text { meses de } \\
\text { duración): Primarias (neuropatías, miopatías y mesenquimopatias). } \\
\text { Secundarias (enfermedades: del colágeno, endocrinas, neurológicas, paraneoplásicas. } \\
\text { asociado a medicamentos, otras.) }\end{array}$ \\
\hline $\begin{array}{l}\text { Obstaculización (tumor polipoide, invaginación, cálculos biliares, cuerpos extraños, } \\
\text { heces). } \\
\text { Lesiones intestinales intrínsecas (estenosis o estrangulaciones: neoplasias, } \\
\text { anastomosis, químicas, enfermedad inflamatoria) } \\
\text { Lesiones extrínsecas (adherencias abdominales: cirugías previas, peritonitis previa, } \\
\text { hernias, neoplasias, vólvulos, carcinomatosis peritoneal, bandas congénitas) } \\
\text { Enfermedad celíaca. }\end{array}$ \\
$\begin{array}{l}\text { Enfermedad de Crohn, atrofia de microvellosidades, enteritis por radioterapia y } \\
\text { quimioterapia, enfermedades congénitas }\end{array}$ \\
\hline Enfermedad extensa de la mucosa del intestino delgado \\
\hline${ }^{(9)}$.
\end{tabular}

dicadores simples de absorción intestinal y equilibrio metabólico.

\section{Diagnóstico en el paciente grave}

Seguidamente al insulto inicial (infección, trauma, cirugía, etcétera) se describen dos fases en la enfermedad crítica $^{(14)}$ :

1. Fase aguda: comprende un período temprano de la fase aguda (antes llamada EBB) de inestabilidad metabólica con aumento importante del catabolismo y razón de la admisión en UCI. Un período tardío de la fase aguda (antes llamada FLOW) más o menos prolongado (3-7 días) donde aún predomina el catabolismo, pérdida de masa muscular y tendencia a estabilizarse los disturbios metabólicos.

2. Fase crónica: Se caracteriza por anabolismo, convalecencia, rehabilitación y en algunos casos un síndrome inflamatorio y catabólico crónico.

En este complejo escenario el paciente puede presentarse sin disfunción ni FI, con disfunción intestinal solamente o fallo intestinal agudo (FIA) tipo I o II (figura 1).

Los test actuales para la evaluación de la disfunción y FI son de valor limitado, los síntomas y signos son muy variados y en ocasiones carecen de objetividad. La definición propuesta por la $\operatorname{ESPEN}^{(8)}$ condiciona el diagnóstico de FI a la presencia simultánea de dos criterios:

1. Disminución en la absorción de macronutrientes y/o agua y electrolitos por alteración en la función intestinal.

2. Necesidad de suplementación IV de macronutrientes y/o agua y electrólitos.

\section{Consideraciones sobre la disminución en la superficie intestinal funcional ${ }^{(15,16)}$}

Es necesario documentar la anatomía y función del intestino restante para diseñar la estrategia nutricional según los segmentos afectados ${ }^{(15)}$ :

1. Yeyuno: el 90\% de la absorción de macronutrientes (carbohidratos, lípidos y aminoácidos) ocurre en los primeros 100 a $150 \mathrm{~cm}$ de intestino proximal, así como la absorción de agua, calcio, fósforo, hierro, ácido fólico y la mayoría de las vitaminas hidrosolubles.

2. Íleon: absorción de vitamina B12 unida al factor intrínseco en los 50-60 cm de íleon distal, vitaminas(A, D, E y K), sales biliares, agua y electrolitos. 


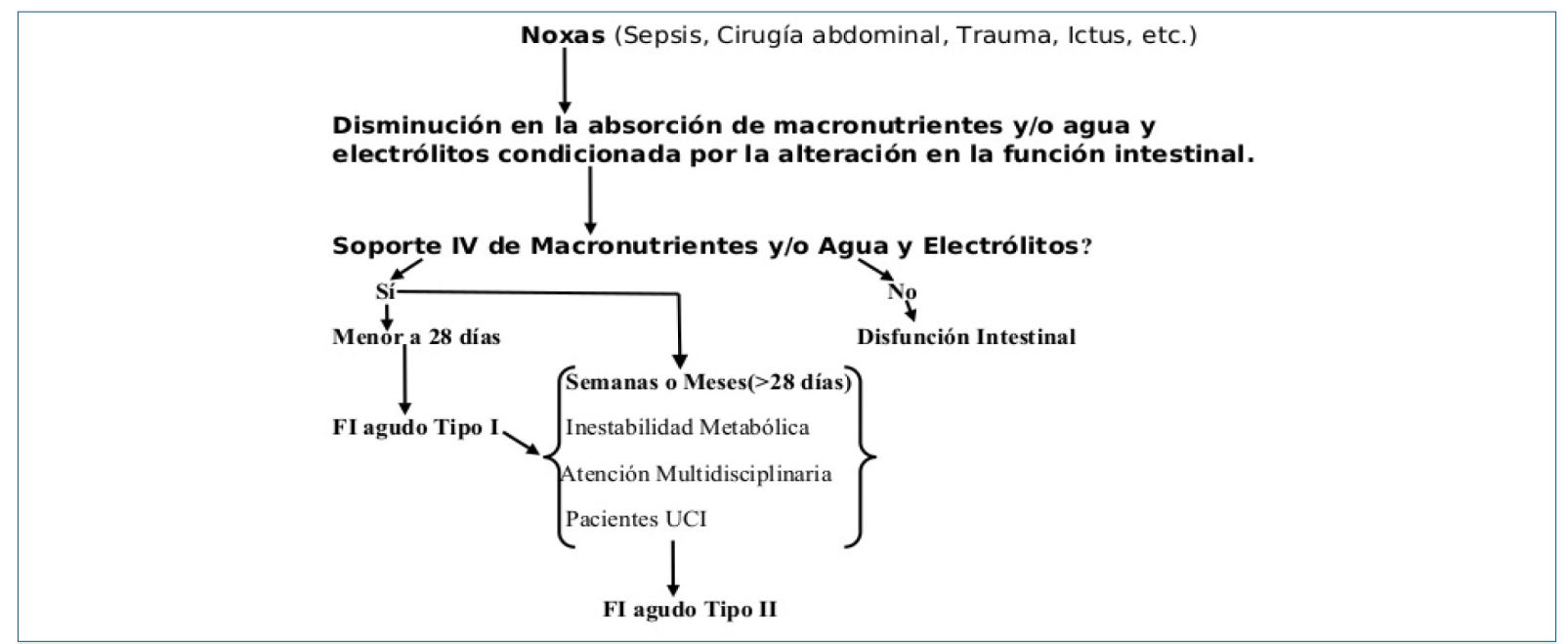

Figura 1. Metodología diagnóstica del FIA y la disfunción intestinal.

3. Colon: absorbe agua en forma más eficaz (puede reabsorber hasta 6 1/día), electrolitos, ácidos grasos de cadena corta y carbohidratos gracias a la flora colónica.

4. Válvula ileocecal: su pérdida reduce el tiempo de tránsito intestinal, alterando la absorción de nutrientes, y es causa de diarrea. Actúa como barrera al paso de bacterias del colon al íleon terminal, que ocasionarían sobrecrecimiento bacteriano alterando la absorción de vitamina $\mathrm{B} 12$, grasas y sales biliares.

\section{Capacidad adaptativa del intestino delgado y colon ${ }^{(15,16)}$}

Intestino delgado: la adaptación es más probable con íleon que con yeyuno. El íleon puede adaptarse en 1-2 años, aumentando longitud y diámetro, ampliación de pliegues de Kerckring y profundización de criptas. Evidencia que se limita a modelos animales sugiere que la hiperplasia de enterocitos y el aumento en la longitud de las vellosidades pueden ocurrir tan pronto como 48 horas después de la resección, mediada por factores tróficos (péptido 2 similar al glucagón, enteroglucagón, factor de crecimiento epidérmico, glutamina, hormona del crecimiento, colecistoquinina, gastrina, neurotensina, leptina y factor de crecimiento similar a la insulina).

Colon: el colon conservado es importante en la digestión, la ralentización de la motilidad intestinal mediada por hormonas y la estimulación de la adaptación intestinal para la reabsorción de agua y electrolitos. También para la absorción de ácidos grasos de cadena corta producidos por la digestión de carbohidratos mediada por bacterias colónicas y la absorción de ácidos grasos de cadena media solubles en agua.

Biomarcadores en la insuficiencia intestinal: en la mayoría de las fallas orgánicas, los biomarcadores séricos constituyen pilares para el diagnóstico, evolución y pronóstico. La cuantificación de la actividad metabólica de la masa enterocitaria representa hipotéticamente la capacidad absortiva de nutrientes en el intestino. En este sentido, dos biomarcadores muestran resultados prometedores: la citrulina plasmática y la apolipoproteína AIV (Apo AIV) ${ }^{(17,18)}$.

1. Citrulina: aminoácido que no se incorpora a proteínas, producido casi exclusivamente por los enterocitos, principalmente del intestino delgado proximal, como parte del metabolismo de la glutamina. Finaliza en los riñones liberando arginina. El valor sérico normal es 40 $\mathrm{mcmol} / \mathrm{l}$ y no se altera por el efecto del primer paso hepático, la dieta o la inflamación ${ }^{(18,19)}$. Crenn y colaboradores ${ }^{(20)}$ demostraron que su valor plasmático se correlaciona con la longitud del intestino delgado como un biomarcador potencial de masa de enterocitos en pacientes con enfermedad celíaca. Cuando los niveles plasmáticos alcanzan $20 \mathrm{mcmol} / \mathrm{l}$ en el contexto de síndrome de intestino corto (SIC), la insuficiencia intestinal permanente es predecible con una sensibilidad del $92 \%$ y especificidad del $90 \%{ }^{(18,19)}$.

2. Apo AIV: en humanos es sintetizada exclusivamente por enterocitos y representa hasta el $4 \%$ de las proteínas sintetizadas por estos. Se expresa en el íleon, estimulando la producción en el yeyuno ${ }^{(18)}$. Un valor de corte de 4,6 mg/100 ml es predictivo de rehabilitación intestinal y establece una diferencia entre los pacientes que alcanzan alimentación oral frente a los que permanecen en nutrición parenteral ${ }^{(21)}$.

\section{Abordaje multidisciplinario del paciente grave con insuficiencia intestinal}

El paciente grave con FI se presenta generalmente con fístula de alto gasto o enterostomía, sepsis y problemas 
Tabla 3. Plasma y fluidos IV más usados.

\begin{tabular}{|c|c|c|c|c|c|c|c|c|c|c|c|}
\hline \multicolumn{7}{|c|}{ Composición y osmolalidad del plasma y cristaloides } & \multirow[t]{2}{*}{ Glucosa } & \multirow[t]{2}{*}{ Lactato } & \multirow[t]{2}{*}{ Acetato } & \multirow[t]{2}{*}{ Gluconato } & \multirow{2}{*}{$\begin{array}{c}\text { Osmola- } \\
\text { lidad } \\
\text { (mOsm/L) }\end{array}$} \\
\hline Fluido & $\mathrm{Na}$ & K & $\mathrm{Cl}$ & $M g$ & $\mathrm{Ca}$ & $\mathrm{HCO}_{3}$ & & & & & \\
\hline Plasma & 142 & 4,3 & 103 & 1,25 & 2,5 & 24 & 0,8 & & & & 291 \\
\hline $0,9 \% \mathrm{NaCl}$ & 154 & 0 & 154 & & & & & & & & 308 \\
\hline $\begin{array}{l}0,18 \% \mathrm{NaCl} / \\
4 \% \text { Dextrosa }\end{array}$ & 30 & 0 & 30 & & & & 40 & & & & 284 \\
\hline $\begin{array}{l}\text { 0,45\% } \mathrm{NaCl} / \\
5 \% \text { Dextrosa }\end{array}$ & 77 & 0 & 77 & & & & 50 & & & & 406 \\
\hline Hartmann & 131 & 5 & 111 & & 2 & & & 29 & & & 278 \\
\hline Ringer/lactato & 130 & 4 & 109 & & 1,5 & & & 28 & & & 273 \\
\hline Plasmalyte 148 & 140 & 5 & 98 & 1,5 & & & & & 27 & 23 & 294 \\
\hline 5\% Dextrosa & 0 & 0 & 0 & & & & 50 & & & & 278 \\
\hline
\end{tabular}

Iones en mmol/l; osmolalidad en mOsm/l; Glucosa g/l.

asociados al SIC (deshidratación, desnutrición, alteraciones hidroelectrolíticas) en el contexto de FIA tipo $\mathrm{II}^{(22)}$.

Dos acrónimos describen la estrategia terapéutica del FIA ${ }^{(22,23)}$ : SNAPP (Sepsis, Nutrición, Anatomía de la fístula, Protección (piel y herida), Plan quirúrgico) y SOWATS (Sepsis, Optimización nutricional, Cuidado de heridas y estomas, Anatomía intestinal y de la fístula, Tiempo y Planificación quirúrgica). Ambos pueden utilizarse en pacientes con FI sin fístulas ${ }^{(22,23)}$.

Resucitación inicial y corrección hidroelectrolítica: los primeros días, hasta uno o dos meses después de la resección intestinal y la formación del estoma/fístula (fase hipersecretora), se caracterizan por importantes pérdidas de líquidos y electrólitos ${ }^{(24)}$. Se reconocen cuatro indicaciones para la terapia con fluidos: resucitación, mantenimiento, reemplazo y redistribución ${ }^{(24,25)}$.

En la tabla 3 se muestran algunas de las soluciones comúnmente usadas y su composición en relación al plasma.

La resucitación tiene como objetivo restaurar y mantener el volumen intravascular para una perfusión tisular adecuada. Para urgencias se recomienda el uso de cristaloides balanceados isotónicos. Como primera opción, la solución de Hartmann o el ringer lactato son adecuadas. Debe evitarse el uso de dextrosa al 5\% como solución para la resucitación.

El cloruro de sodio al 0,9\% es una mala elección en estados de shock con acidosis, potencialmente empeorará la situación (causa acidosis hiperclorémica), tiene un contenido de sodio suprafisiológico y no es isotónico.
Los fluidos de mantenimiento deben cubrir las necesidades basales diarias de agua y electrolitos en casos que estos no puedan ser administrados por vía enteral. Los fluidos para el mantenimiento IV en adultos deben incluir:

- Agua $25-30 \mathrm{ml} / \mathrm{kg} / \mathrm{día}$.

- Potasio, sodio y cloro: $1 \mathrm{mmol} / \mathrm{kg} /$ día.

- Glucosa: 50-100 g/día

Los fluidos de reemplazo son diseñados para sustituir pérdidas más específicas: estomas, fístulas, fiebre, heridas abiertas, etcétera. Suelen usarse cristaloides balanceados e isotónicos. Pautas recientes ${ }^{(24)}$ recomiendan igualar la cantidad de líquidos y electrolitos tan cerca como sea posible a la del fluido perdido. En el caso de fístulas/enterostomías, los fluidos IV más usados para el reemplazo son la solución de Ringer lactato o Hartmann. Cuando el déficit se debe a una pérdida de cloruro del fluido gástrico se puede elegir solución salina al $0,9 \%$. Es necesario corregir bajas concentraciones séricas de potasio, magnesio y fosfato: asociadas a alteraciones de la motilidad intestinal y desarrollo del síndrome de realimentación.

\section{Fases de la fluidoterapia(24,25)}

- Fase de rescate o resucitación (0 - primeras horas). Rescate del paciente mediante un manejo temprano $\mathrm{y}$ adecuado de fluidos (early adequate fluid management $[\mathrm{EAFM}])$. Debe personalizarse según predictores dinámicos de la relación precarga/gasto 
cardíaco o respuesta a bolus de fluidos (3-4 ml/ $\mathrm{kg}$ en 10-15 min).

- Fase de optimización (24 h-72 h). Sustituir las pérdidas y evitar la sobrecarga de fluidos (que conduce a disfunción orgánica e hipertensión abdominal). El balance acumulado de fluidos constituye un marcador pronóstico durante la primera semana de estadía en UCI y si es mayor a $10 \%$ se asocia a aumento de la mortalidad.

- Fase de estabilización (tercer-cuarto día). Evitar la hipovolemia y sobrecarga de fluidos: considerar en casos sobrerresucitados al inicio el manejo conservador tardío de fluidos (late conservative fluid management [LCFM]), definido como dos balances de fluidos negativos consecutivos durante la primera semana.

- Fase de evacuación (semanas): conservar la estabilidad hemodinámica, mientras se resuelve la sobrecarga secundaria a la fluidoterapia inicial, mantener la normovolemia y las necesidades básicas. Considerar en caso de sobrecarga de fluidos la desresucitación (late goal directed fluid removal [LGFR]) mediante el uso de diuréticos o ultrafiltración en algunos casos.

\section{Predicción de la respuesta(24,25)}

Consiste en predecir -antes de la administración de líquidos- si ésta será beneficiosa:

- Evitar marcadores estáticos de precarga cardíaca, como la presión venosa central (PVC) o la presión de oclusión de la arteria pulmonar (PAOP).

- Se recomienda evaluación dinámica de la relación precarga/gasto cardíaco. Por ejemplo: variabilidad de presión de pulso (VPP) y del volumen sistólico (VVS), índice de variabilidad pletismográfica (IVP) y de perfusión (IP), elevación pasiva de piernas/aumento del gasto cardíaco medido (PiCCO, volume view, ecocardiograma), índice de colapso de vena cava inferior (VCI) sin ventilación mecánica (VM) o de distensibilidad de VCI con VM.

\section{Monitorización $(24,25)$}

- Parámetros clínicos: diuresis, presión arterial y presión arterial media (PAM), PIA, llenado capilar, frecuencia cardiaca, balance diario y acumulado de fluidos, peso, auscultación cardiaca y pulmonar, presencia de edemas.

- Laboratorio: lactato, BUN, ionograma, gasometría, creatinina, hematocrito, saturación venosa central de oxigeno $\left(\mathrm{SvcO}_{2}\right)$, albúmina, osmolalidad sérica.
- Imagen: radiografía de tórax, ecografías (cardiaca, pulmonar, venosa (VCI, yugular interna), abdominal).

- Hemodinámica: PiCCO y PiCCO plus, IVP, IP, entre otros.

Prevención y tratamiento de la sepsis: la sepsis puede originarse en la cavidad abdominal, ocurrir por translocación bacteriana (por ejemplo: casos de distensión intestinal grave, isquemia intestinal subaguda sin perforación), producto de intervenciones y procederes terapéuticos: bacteriemia relacionada al catéter venoso central (BRCVC) o causas extraabdominales (neumonía, infección del tracto urinario), presentándose con un amplio espectro de síntomas y signos, y es la principal causa de muerte en pacientes con FIA ${ }^{(26)}$. En pacientes con fístula enterocutánea la sepsis intraabdominal se asocia al 77\% de la mortalidad ${ }^{(27)}$. En una colección intraabdominal, se recomienda un abordaje mínimamente invasivo, realizándose drenaje percutáneo o de no ser posible, por vía alternativa (por ejemplo transgástrica, transrrectal o transvaginal), convenientemente guiado por tomografía computadorizada (TC) o ecografía, que además permite tomar muestras para estudios microbiológicos y tratamiento antimicrobiano dirigido. La reintervención para el control de foco séptico intraabdominal debe reservarse para situaciones en las que fallan los enfoques mínimamente invasivos ${ }^{(26,28)}$.

Nutrición: la NE previene la atrofia de la mucosa, contribuye a preservar la microbiota y a reducir la incidencia de bacteriemia y la mortalidad ${ }^{(26)}$. Los esfuerzos para iniciarla deben comenzar una vez que el paciente esté euvolémico, hemodinámica y metabólicamente estable ${ }^{(26,28)}$.

La Sociedad de Medicina Crítica (Society of Critical Care Medicine [SCCM]) y la Sociedad Americana para la Nutrición Enteral y Parenteral (American Society for Parenteral and Enteral Nutrition [ASPEN]) ${ }^{(29)}$ y la $\operatorname{ESPEN}^{(14)}$ recomiendan iniciar la NE en forma temprana (primeras $48 \mathrm{~h}$ ) en pacientes graves cuando la vía oral no es posible.

Según la ESPEN ${ }^{(14)}$ no se debe iniciar la NE completa (más del 70\% del gasto energético en reposo [GER], pero menos del $100 \%$ ) en forma temprana en pacientes críticos, y se prescribirá entre el tercero y el séptimo días. Mientras la ASPEN/SCCM ${ }^{(29)}$ recomienda evitar la NE completa solamente en pacientes que tengan un riesgo nutricional elevado (Nutritional Risk Screening (NRS-2002) $\geq 5$ o Nutrition Risk in the Critically Ill (NUTRIC) $\geq 5$, sin interleucina 6) o gravemente desnutridos, avanzando para proporcionar $>80 \%$ de la energía y proteínas estimadas en 48-72 horas durante la primera semana de hospitalización. 
En pacientes con fístulas o estomas de alto gasto y acceso distal posible debe considerarse la reinfusión de quimo (enteroclisis) ${ }^{(14,29,30)}$.

\section{Cuándo iniciar la nutrición parenteral (NP)}

\section{A) ASPEN/SCCM(29)}

- Evaluar riesgo nutricional de los pacientes al ingreso en UCI (por ejemplo NRS-2002, NUTRIC).

- Evitar NP durante los primeros 7 días en UCI en el paciente con bajo riesgo nutricional (por ejemplo NRS $2002 \leq 3$ o NUTRIC $\leq 5$ ), que no pueda mantener la ingesta voluntaria y la NE no sea factible.

- Iniciar NP exclusiva lo antes posible después de la admisión en la UCI, en el paciente con alto riesgo nutricional (por ejemplo NRS $2002 \geq 5$, NUTRIC $\geq 5$ ) o con desnutrición grave, cuando la NE no es factible.

\section{B) $E S P E N^{(14)}$}

- Todo paciente crítico que permanezca más de $48 \mathrm{~h}$ en UCI debe considerarse en riesgo de desnutrición.

- En caso de contraindicaciones para la vía oral y NE, la NP debe ser implementada entre el $3^{\text {er }}$ y $7^{\circ}$ día de estadía en UCI.

\section{Cuándo iniciar la NP como suplemento de la NE}

ESPEN, ASPEN/SCCM ${ }^{(14,29)}$ : la NP no debe iniciarse hasta que todas las estrategias para maximizar la tolerancia de la NE se han intentado.

ESPEN $^{(14)}$ : pacientes que no toleran la dosis completa de NE durante la primera semana en la UCI, deben evaluarse individualmente en relación a la seguridad y beneficios de iniciar la NP.

ASPEN/SCCM ${ }^{(29)}$ : independientemente del riesgo nutricional, el uso de NP suplementaria debe considerarse solo después de siete a diez días si la NE no puede satisfacer $>60 \%$ de las necesidades de energía y proteínas.

El papel de la NP suplementaria queda por definir en términos de tiempo, cantidad y composición. Casaer y colaboradores $^{(31)}$ observaron que la NP temprana (complementaria o exclusiva) se asocia con un aumento de la morbilidad, estadía prolongada en UCI, mayor dependencia de la ventilación mecánica, aumento en la tasa de infección y necesidad de terapia de reemplazo renal. Sin embargo, otros estudios que abordan la NP suplementaria $^{(32,33)}$ no mostraron hallazgos similares. El estudio EAT-ICU $^{(34)}$ sobre asociar la NP suplementaria con la alimentación enteral desde el ingreso en UCI con un objetivo definido por calorimetría, no encontró daño o ventaja en términos de morbilidad, función a largo plazo o mortalidad.
En situaciones de alto gasto (estomas con pérdidas mayores a $1.500 \mathrm{ml} /$ día) los esfuerzos deben centrarse en implementar una dieta con alto contenido calórico y baja en fibra que pase lentamente por el intestino delgado, aumentando la absorción de nutrientes, evitar fluidos hipotónicos vía oral o enteral, restringiendo el volumen de fluidos: $500-1000 \mathrm{ml} / 24 \mathrm{~h}^{(28,35)}$. Usar soluciones, como la de rehidratación oral de la OMS o la solución de St Mark's, para reemplazar las pérdidas de electrólitos. Estas se componen de 3,5 g de cloruro de sodio, $2,5 \mathrm{~g}$ de bicarbonato de sodio, $20 \mathrm{~g}$ de glucosa en $11 \mathrm{de}$ agua ${ }^{(23)}$. Se puede administrar magnesio oral además de las soluciones de electrólitos cuando sea necesario. Se requiere un cuidadoso balance mientras se titula entre la suplementación oral y parenteral ${ }^{(26,28)}$.

Es recomendable probar NE si el gasto de la fístula/estoma es $<1,51$. Las fórmulas semielementales reducen significativamente el gasto de la fístula ${ }^{(28)}$. La NE puede estar contraindicada: cuando la longitud intestinal es insuficiente $(<75 \mathrm{~cm})$, si existe discontinuidad intestinal, aumento $>3.000 \mathrm{ml}$ en la salida de la fístula/estoma con el inicio de NE, intolerancia a NE, cuando el acceso enteral no se puede establecer o mantener ${ }^{(28,35)}$.

\section{Farmacoterapia en situaciones de alto gasto}

En la fase hipersecretora es común el uso de medicamentos inhibidores de la bomba de protones o antagonistas de los receptores $\mathrm{H}_{2}$ de histamina y antidiarreicos como la loperamida o codeína para disminuir el gasto la de la fístula o estoma ${ }^{(35)}$, en un centro de insuficiencia intestinal británico, altas dosis de loperamida (40 $\mathrm{mg} /$ día) y codeína ( $240 \mathrm{mg} /$ día) se utilizaron para controlar el gasto de fístulas refractarias con éxito ${ }^{(36)}$.

La evidencia sobre los beneficios de la somatostatina y su análogo sintético octreótido en la enterostomía de alto gasto es controversial, su uso en una unidad dedicada al FI se limita a pruebas de $72 \mathrm{~h}$ y en caso de disminuir el débito del estoma o fístula continuaría aplicándo$\mathrm{se}^{(36)}$.

En la tabla 4 se resumen los fármacos más utilizados, dosis, consideraciones sobre su aplicación y efectos adversos.

\section{Sobre teduglutide}

Es un análogo sintético del péptido-2, similar al glucagón, con mayor vida media, y representa el primer abordaje no sintomático del SIC ${ }^{(38)}$.

Fue aprobada para el uso en pacientes adultos con SIC en Estados Unidos por la FDA y en la Unión Europea por la EMA en 2012. La aprobación se basó en los resultados de un estudio en fase III de 24 semanas, doble ciego, controlado con placebo (STEPS) ${ }^{(39)}$ : pacientes con FI por SIC dependientes de NP, recibieron 0,05 
Tabla 4. Fármacos en el fallo intestinal.

\begin{tabular}{|c|c|c|c|}
\hline Fármaco & Dosis & Efectos adversos & Otras consideraciones \\
\hline \multicolumn{4}{|c|}{ Efecto antidiarreico por reducción del tránsito intestinal } \\
\hline Loperamida & $2 \mathrm{mg}$ oral hasta 16 veces/día & $\begin{array}{l}\text { Distensión abdominal, íleo paralitico, } \\
\text { somnolencia, dispepsia, eritema. } \\
\text { angioedema, megacolon tóxico. }\end{array}$ & $\begin{array}{l}\text { Puede ser necesaria dosis mayores en } \\
\text { pacientes con anastomosis yeyunocólica o } \\
\text { yeyunostomía debido a circulación } \\
\text { enterohepática alterada y menor absorción } \\
\text { de loperamida }\end{array}$ \\
\hline Difenoxilato-atropina & $2,5-7,5 \mathrm{mg}$ oral 4 veces/día & $\begin{array}{l}\text { Sedación / somnolencia, confusión, } \\
\text { euforia, depresión, náuseas,vómitos, } \\
\text { malestar abdominal, megacolon tóxico e } \\
\text { íleo paralítico. }\end{array}$ & Evitar dosis mayores de $30 \mathrm{mg} / \mathrm{día}$ \\
\hline Codeína & $30-60 \mathrm{mg} 4$ veces/día & $\begin{array}{l}\text { Constipación, náuseas, vómitos, disnea, } \\
\text { íleo paralitico, depresión circulatoria y } \\
\text { respiratoria, sedación, somnolencia }\end{array}$ & Dependencia y abstinencia \\
\hline Colestiramina & $<24 \mathrm{~g}$ oral/día & $\begin{array}{l}\text { Constipación, dispepsia, náuseas y } \\
\text { vómitos. }\end{array}$ & $\begin{array}{l}\text { En pacientes con resección de íleon }>100 \\
\mathrm{~cm} \text {, la colestiramina puede no ser } \\
\text { beneficiosa: superficie insuficiente para la } \\
\text { absorción de sales biliares. }\end{array}$ \\
\hline \multicolumn{4}{|c|}{ Efecto antidiarreico por reducción de las secreciones gastrointestinales } \\
\hline $\begin{array}{l}\text { Inhibidores de la } \\
\text { bomba de protones } \\
\text { (IBP) por ejemplo. } \\
\text { omeprazol }\end{array}$ & $40 \mathrm{mg}$ oral $2-3$ veces/día & $\begin{array}{l}\text { Cefalea, dolor abdominal, náuseas, } \\
\text { vómitos, flatulencia }\end{array}$ & $\begin{array}{l}\text { Reduce la secreción gástrica y puede } \\
\text { aumentar la absorción de agua intestinal. } \\
\text { Los antagonistas de receptores de } \mathrm{H} 2 \\
\text { también reducen la secreción de gástrica, } \\
\text { pero pueden no ser tan efectivos como los } \\
\text { IBP. }\end{array}$ \\
\hline Octreótido & $\begin{array}{l}\text { Hasta } 500 \mathrm{mg} \mathrm{SC} 3 \text { veces al día (o dosis } \\
\text { IV equivalente) }\end{array}$ & $\begin{array}{l}\text { Se han notificado: hipoglucemia, mareos, } \\
\text { rubor facial transitorio y náuseas tras una } \\
\text { sobredosis de octreótido. }\end{array}$ & $\begin{array}{l}\text { Considerar si se requieren }>3 \mathrm{~L} \text { de líquido } \\
\text { IV y diarrea o malabsorción refractaria a } \\
\text { otros medicamentos. }\end{array}$ \\
\hline Clonidina & $\begin{array}{l}0,1-0,2 \mathrm{mg} \text { oral } 2 \text { veces/día (o equivalente } \\
\text { transdermica) }\end{array}$ & $\begin{array}{l}\text { Hipotensión, prurito, constipación, tinnitus, } \\
\text { vértigo, arritmias, bloqueo AV. }\end{array}$ & $\begin{array}{l}\text { Considerar si yeyunostomía y débito } \\
\text { importante refractario a otros } \\
\text { medicamentos }\end{array}$ \\
\hline \multicolumn{4}{|c|}{ Medicamentos que promueven la adaptación intestinal } \\
\hline Teduglutide & $0,05 \mathrm{mg} / \mathrm{kg} \mathrm{SC}$ una vez al día & $\begin{array}{l}\text { Dolor y distensión abdominal, náuseas, } \\
\text { enrojecimiento en el lugar de la inyección, } \\
\text { vómitos, sobrecarga de líquidos e } \\
\text { hipersensibilidad. Otros incluyen: } \\
\text { obstrucción intestinal, enfermedad biliar y } \\
\text { pancreática, insuficiencia cardíaca } \\
\text { congestiva. } \\
\text { Posible crecimiento de neoplasias } \\
\text { existentes(basado en su actividad } \\
\text { farmacológica en animales). }\end{array}$ & $\begin{array}{l}\text { Durante la evaluación clínica, la dosis } \\
\text { máxima estudiada fue de } 80 \text { mg/día } \\
\text { durante } 8 \text { días. La adaptación para } \\
\text { restaurar algunas funciones ocurre } \\
\text { típicamente dentro de los } 2 \text { años de la } \\
\text { resección, pero considere incluso después } \\
\text { de } 2 \text { años. } \\
\text { No se ha establecido una dosis tóxica. } \\
\text { Puede aumentar la absorción de } \\
\text { medicamentos orales; requiriendo } \\
\text { reducción de la dosis del fármaco } \\
\text { concomitante. Evitar si malignidad actual o } \\
\text { antecedentes de malignidad en los últimos } \\
5 \text { años. } \\
\text { Se recomienda una colonoscopía de } \\
\text { seguimiento (o imágenes alternativas) al } \\
\text { final de } 1 \text { año de tratamiento. }\end{array}$ \\
\hline $\begin{array}{l}\text { Hormona de } \\
\text { crecimiento humana } \\
\text { recombinante }\end{array}$ & $0,13 \mathrm{mg} / \mathrm{kg} \mathrm{SC} /$ día por 12 semanas & \multicolumn{2}{|c|}{$\begin{array}{l}\text { Basado en pequeño ensayo clínico(37): se reporta un incremento en la nutrición } \\
\text { enteral del } 54 \% \text { en } 8 \text { niños con síndrome de intestino corto neonatal en nutrición } \\
\text { parenteral a largo plazo. } \\
\text { Aumenta el factor de crecimiento similar a la insulina (IGF-1) y puede aumentar el } \\
\text { peso corporal. }\end{array}$} \\
\hline
\end{tabular}


$\mathrm{mg} / \mathrm{kg}$ de teduglutide o placebo $1 \mathrm{vez} /$ día durante 24 semanas. La proporción de pacientes que consiguieron una reducción $\geq 20 \%$ en el volumen de NP en la semana 20 frente al valor basal y que mantuvieron dicha respuesta en la semana 24 (respondedores) fue significativamente mayor en el grupo de teduglutida que en el de placebo $(63 \%$ vs. 30\%; p =0,002). La reducción absoluta media frente al valor basal del volumen de NP en la semana 24 fue significativamente mayor (4,4 vs. 2,3 1/semana; $<<0,001)$ y se mantuvo a largo plazo. En la extensión abierta del estudio STEPS (STEPS-2) ${ }^{(40)}$, todos los pacientes continuaron el tratamiento con teduglutide o fueron cambiados a él $(\mathrm{n}=88)$, durante 2 años adicionales y 65 (74\%) completaron el estudio, 13 pacientes lograron autonomía enteral total y la mayoría de los pacientes alcanzaron o mantuvieron respuestas importantes con la teduglutide con las mayores reducciones en la NP en el subgrupo de mayor exposición a teduglutide.

En la primera serie pediátrica argentina tratada con teduglutide $^{(41)}$ de 62 niños con síndrome de intestino corto, 5 recibieron teduglutide $0,05 \mathrm{mg} / \mathrm{kg} /$ día $\mathrm{SC}$. El aporte de nutrición parenteral se redujo $\pm 48 \%$, pudiendo suspenderse en 2 (a las semanas 29 y 24 del tratamiento).

\section{Protección de la piel que rodea la fístula/estoma y cuidado de la herida}

El aporte de la enfermera especialista en ostomía es fundamental para la protección y cuidado de la piel perifístula y herida ${ }^{(23,28)}$. El contenido procedente de la porción proximal del intestino delgado puede causar excoriación cutánea en menos de $3 \mathrm{~h}^{(36)}$. Los objetivos en el cuidado de la herida y drenaje de la fístula o estoma son: estabilizar y controlar el drenaje, evitar pérdida de la piel y tejidos circundantes, minimizar el dolor y aislamiento social, contribuir al cierre de la herída o fístu$\mathrm{la}^{(35)}$. Las fístulas de gasto moderado pueden manipularse con bolsa de ostomía y protección adecuada de la piel alrededor en forma de anillo adhesivo, pasta, polvo o apósito hidrofílico ${ }^{(28,35)}$. La terapia con presión negativa tópica (TPN) es la aplicación de la presión negativa a través de la herida o fístula para ayudar en su cicatrización y estabilizar el drenaje ${ }^{(42)}$, el cierre asistido por vacío (VAC) de KCI Medical, San Antonio, Texas, ha mostrado resultados exitosos en algunas series de casos sin constituir nivel I de evidencia ${ }^{(42,43)}$.

\section{Anatomía}

El mapeo anatómico ayuda a comprender la ubicación y longitud del intestino restante y predecir resultados posoperatorios, incluida la necesidad de nutrición parenteral a largo plazo. Investigaciones radiológicas, como: TC y resonancia magnética (RM), fluoroscopía, estu- dios de tubo o drenaje y fístulogramas o sinogramas pueden ser necesarios para delimitar el origen, destino y número de fístulas. La evaluación endoscópica a través de un orificio natural, estoma o fístula también puede ser útil como complemento para la planificación preoperatoria y raramente proporcionar terapia con stent o cierre con $\operatorname{clip}^{(23,28,35)}$. Estos deben realizarse al menos 7 a 10 días después de la reanimación con líquidos y electrólitos, control de infecciones y tratamiento de heri$\operatorname{das}^{(28)}$.

\section{Plan y cirugía definitiva}

Aproximadamente el 30\% de los pacientes experimentarán curación de la fístula entre las 4 y 8 semanas después de la provisión de nutrición, control de sepsis y drenaje de la fístula ${ }^{(44)}$. Si la curación no ocurre dentro de este período, deben iniciarse los planes para la cirugía definitiva. La reparación quirúrgica definitiva debe retrasarse durante un mínimo de 6 meses desde la última intervención quirúrgica. Dado que las adherencias tempranas tienden a ser más tenaces 10 días a 3 meses después de la laparotomía, la reintervención quirúrgica temprana ( $<3$ meses desde última cirugía abierta) está asociada con altas tasas de mortalidad, morbilidad y riesgos de recurrencia ${ }^{(28)}$, realizándose sólo en situaciones que ponen en peligro la vida, entre éstas ${ }^{(23,35)}$ :

- Drenaje de focos sépticos cuando esto no puede ser logrado por vía percutánea.

- Extirpación de intestino isquémico.

- Abrir cavidades de abscesos de la pared abdominal.

- Construcción de una estoma de derivación proximal en raras circunstancias.

- Falla anastomótica catastrófica no controlada.

En la figura 2 se muestra un protocolo aplicado al paciente grave con fallo intestinal y la intervención multidisciplinaria según las etapas.

\section{Las unidades dedicadas}

Se trata de unidades especializadas en insuficiencia intestinal. Los principales objetivos son: proporcionar un cuidado experto para un soporte IV seguro y cuidado del catéter para minimizar las tasas de BRCVC, mantener un equilibrio de líquidos preciso, cuidados de estomas y heridas, alimentación distal y atención psicológica. Cuentan con enfermeras especializadas y una gama completa de especialistas disponibles: intensivistas, gastroenterólogos, cirujanos, dietistas, farmacéuticos, psicólogos/psiquiatras y radiólogos intervencionistas. Existe evidencia de que en estas unidades se reducen las tasas de complicaciones y la mortalidad hasta en un $50 \%{ }^{(22)}$. 


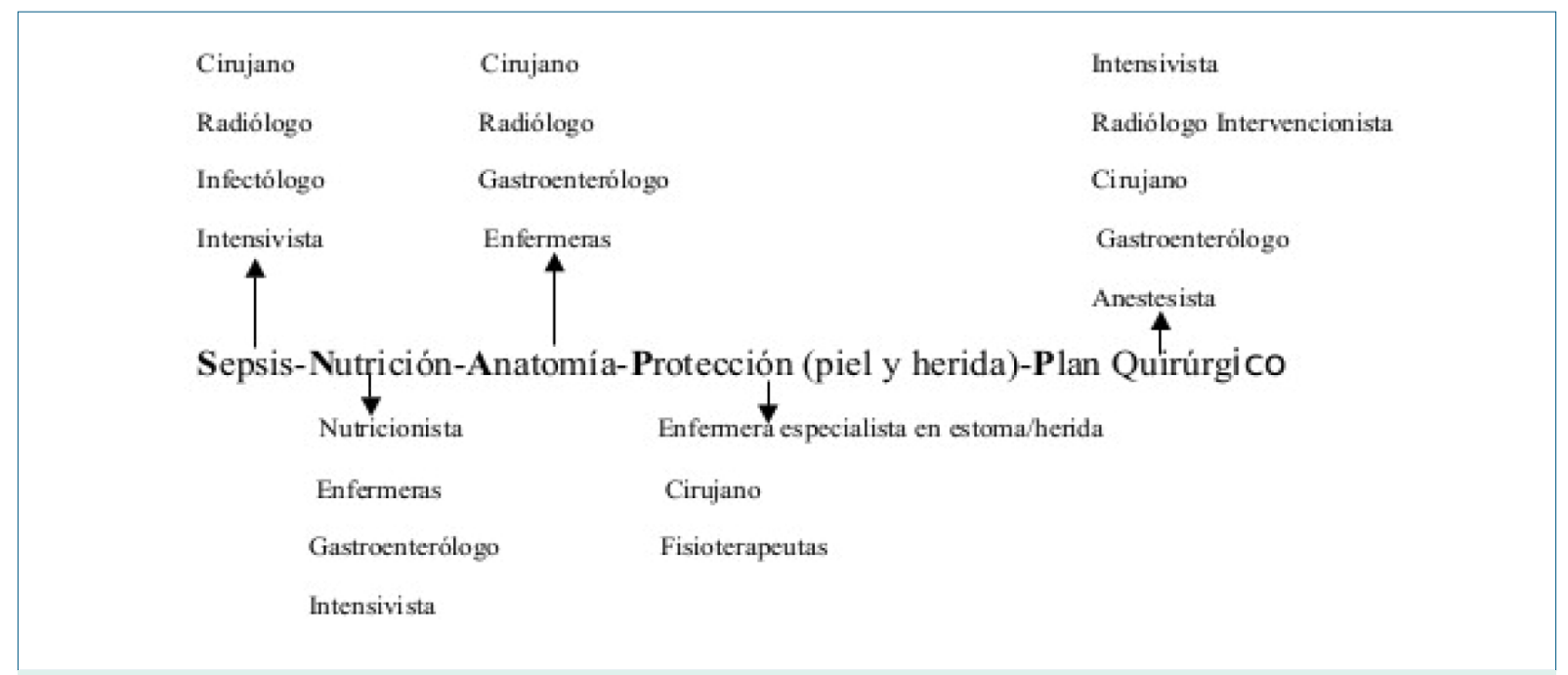

Figura 2. Protocolo SNAPP en el FI. Abordaje multidisciplinario.

\section{Conclusiones}

Las alteraciones gastrointestinales son frecuentes en el paciente crítico y un determinante en su mortalidad. Reconocer el fallo y disfunción intestinal permite optimizar el tratamiento, reducir la mortalidad e incidencia de complicaciones, las unidades dedicadas con un staff de experiencia en el manejo del FIA constituyen un aspecto clave en este sentido.

\section{Summary}

Introduction: in critically ill patients, intestine function expresses the response to stress. Symptoms of intestinal failure in intensive care units are not specific and are not included in commonly used severity scales. However, $62 \%$ of critically ill patients present gastrointestinal symptoms at least once a day, demonstrating the relationship between the appearance of gastrointestinal problems and mortality in critical patients.

Objective: to provide the necessary theoretical knowledge about intestinal failure as an exclusive entity and how to recognize it in critically ill patients, classification and a multidisciplinary approach to treatment of this condition.

Methods: a qualitative and systematic review was carried out of the literature published and updated until February 2020 both in English and in Spanish.

Conclusions: gastrointestinal disorders are frequent in critically ill patients and constitute a determining factor in their mortality. Recognizing intestinal failure and dysfunction allows the optimization of treatment, reduces mortality and the incidence of complications. The creation of dedicated units with experienced staff in the management of acute intestinal failure is a key aspect in this regard.

\section{Resumo}

Introdução: em pacientes críticos, o intestino é o protagonista da resposta ao estresse. Os sintomas de insuficiência intestinal em unidades de terapia intensiva não são específicos e não estão incluídos nas escalas de gravidade comumente usadas; no entanto, $62 \%$ dos pacientes criticamente enfermos apresentam sintomas gastrointestinais pelo menos uma vez ao dia, demonstrando a relação entre o aparecimento de problemas gastrointestinais e mortalidade em pacientes críticos.

Objetivo: proporcionar o conhecimento teórico necessário sobre a insuficiência intestinal como entidade exclusiva, seu reconhecimento em pacientes críticos, classificação e abordagem multidisciplinar do tratamento.

Métodos: foi realizada uma revisão qualitativa e sistemática nos idiomas inglês e espanhol da literatura publicada e atualizada até fevereiro de 2020.

Conclusões: as alterações gastrointestinais são frequentes em pacientes críticos e determinam sua mortalidade. O reconhecimento da insuficiência e disfunção intestinal permite otimizar o tratamento, reduzindo a mortalidade e a incidência de complicações; contar com unidades dedicadas e com equipe experiente no manejo da insuficiência intestinal aguda são um aspecto fundamental nesse sentido.

\section{Bibliografía}

1. Sánchez AC, Espinosa BL, Martínez LAF. Fallo gastrointestinal agudo en el paciente crítico. Nutr Clín Med 2017; 11(2):59-73.Disponible en: http://www.aulamedica.es/nutricionclinicamedicina/pdf/5050.pdf (Consulta: 26 octubre 2019).

2. Salvo-Romero E, Alonso Cotoner C, Pardo Camacho C, Casado Bedmar M, Vicario M. The intestinal barrier function and its involvement in digestive disease. Rev Esp Enferm 
Dig 2015; 107(11):686-96. Disponible en: http://scielo.isciii.es/scielo.php?script=sci_art-

text\&pid=S1130-1082015001100007\&lng=es (Consulta: 16 febrero 2019).

3. Andoh A. Physiological role of gut microbiota for maintaining human health. Digestion 2016; 93(3):176-81.

4. Berg G, Rybakova D, Fischer F, Cernava T, Vergès M-CC, Charles T, et al. Microbiome definition re-visited: old concepts and new challenges. Microbiome 2020; 8:103.

5. Klingensmith NJ, Coopersmith $\mathbf{C M}$. The gut as the motor of multiple organ dysfunction in critical illness. Crit Care Clin 2016; 32(2):203-12.

6. Ritz MA, Fraser R, Tam W, Dent J. Impacts and patterns of disturbed gastrointestinal function in critically ill patients. Am J Gastroenterol 2000; 95(11):3044-52.

7. Reintam Blaser A, Poeze M, Malbrain ML, Björck M, Oudemans-van Straaten HM, Starkopf J; Gastro-Intestinal Failure Trial Group. Gastrointestinal symptoms during the first week of intensive care are associated with poor outcome: a prospective multicentre study. Intensive Care Med 2013; 39(5):899-909.

8. Pironi L, Arends J, Baxter J, Bozzetti F, Peláez RB, Cuerda $\mathrm{C}$, et al; Home Artificial Nutrition \& Chronic Intestinal Failure; Acute Intestinal Failure Special Interest Groups of ESPEN. ESPEN endorsed recommendations. Definition and classification intestinal of failure in adults. Clin Nut 2015; 34:171-80.

9. Pironi L, Arends J, Bozzetti F, Cuerda C, Gillanders L, Jeppesen PB, et al. ESPEN guidelines on chronic intestinal failure in adults. Clin Nutr 2016; 35:247-307.

10. Lal S, Teubner A, Shaffer JL. Review article: intestinal failure. Aliment Pharmacol Ther 2006; 24:19-31.

11. Reintam Blaser AR, Malbrain ML, Starkopf J, Fruhwald S, De Waele J, Braun JP. Gastrointestinal function in intensive care patients: terminology, definitions and management. Recommendations of the ESICM working group on abdominal problems. Intensive Care Med 2012; 38(3):384-94.

12. Balogh ZJ, Lumsdaine W, Moore EE, Moore FA. Postinjury abdominal compartment syndrome: from recognition to prevention. Lancet 2014; 384(9952):1466-75.

13. Kirkpatrick AW, Roberts DJ, De Waele J, Jaeschke R, Malbrain ML, De Keulenaer B, et al; Pediatric Guidelines Sub-Committee for the World Society of the Abdominal Compartment Syndrome. Intra-abdominal hypertension and the abdominal compartment syndrome: updated consensus definitions and clinical practice guidelines from the World Society of the Abdominal Compartment Syndrome. Intensive Care Med 2013; 39(7):1190-206.

14. Singer P, Blaser AR, Berger MM, Alhazzani W, Calder PC, Casaer MP, et al. ESPEN guideline on clinical nutrition in the intensive care unit. Clin Nutr 2019; 38(1):48-79.

15. Iyer KR. Surgical management of short bowel syndrome. JPEN J Parenter Enteral Nutr 2014; 38(1 Suppl):53S-59S.

16. Bechtold ML, McClave SA, Palmer LB, Nguyen DL, Urben LM, Martindale RG, et al. The pharmacologic treat- ment of short bowel syndrome: new tricks and novel agents. Curr Gastroenterol Rep 2014; 16(7):392.

17. Crenn P, Messing B, Cynober L. Citrulline as a biomarker of intestinal failure due to enterocyte mass reduction. Clin Nutr 2008; 27(3):328-39.

18. López-Tejero MD, Virgili N, Targarona J, Ruiz J, García $\mathbf{N}$, Oró $\mathbf{D}$, et al. Apo AIV and citrulline plasma concentrations in short bowel syndrome patients: the influence of short bowel anatomy. PLoS One 2016; 11(9):e0163762.

19. Barzal JA, Szczylik C, Rzepecki P, Jaworska M, Anuszewska E. Plasma citrulline level as a biomarker for cancer therapy-induced small bowel mucosal damage. Acta Biochim Pol 2014; 61(4):615-31.

20. Crenn P, Coudray-Lucas C, Thuillier F, Cynober L, Messing B. Postabsorptive plasma citrulline concentration is a marker of absorptive enterocyte mass and intestinal failure in humans. Gastroenterology 2000; 119(6):1496-505.

21. Cadena ME, Vinck EE, Santivañez JJ, Gómez AV. Biomarkers for intestinal failure in short bowel syndrome: a new era in GI rehabilitation?. Rev Colomb Cir 2019; 34(3):277-82.Disponible en: http://www.scielo.org.co/scielo.php?script $=$ sci_arttext\&pid=S2011-75822019000300277\&lng=en (Consulta: 16 febrero 2020).

22. Klek S, Forbes A, Gabe S, Holst M, Wanten G, Irtun O, et al. Management of acute intestinal failure: a position paper from the european society for clinical nutrition and metabolism (ESPEN) Special Interest Group. Clin Nutr 2016; 35(6):1209-18.

23. Adaba F, Vaizey CJ, Warusavitarne J. Management of intestinal failure: the high-output enterostomy and enterocutaneous fistula. Clin Colon Rectal Surg 2017; 30(3):215-22.

24. Malbrain ML, Langer T, Annane D, Gattinoni L, Elbers P, Hahn RG, et al. Intravenous fluid therapy in the perioperative and critical care setting: executive summary of the International Fluid Academy (IFA). Ann Intensive Care 2020; 24:10(1):64. doi: 10.1186/s13613-020-00679-3 .

25. Malbrain ML, Van Regenmortel N, Saugel B, De Tavernier B, Van Gaal PJ, Joannes-Boyau O, et al. Principles of fluid management and stewardship in septic shock: it is time to consider the four D's and the four phases of fluid therapy. Ann Intensive Care. 2018; 8(1):66

26. Pironi L, Corcos O, Forbes A, Holst M, Joly F, Jonkers C, et al; ESPEN Acute and Chronic Intestinal Failure Special Interest Groups. intestinal failure in adults: recommendations from the ESPEN expert groups. Clin Nutr 2018; $37(6$ Pt A):1798-1809.

27. Campos AC, Andrade DF, Campos GM, Matias JE, Coelho JC. An multivariate model to determine prognostic factors in gastrointestinal fistulas. J Am Coll Surg 1999; 188(5):48390.

28. Gribovskaja-Rupp I, Melton GB. Enterocutaneous fistula: proven strategies and updates. Clin Colon Rectal Surg 2016; 29(2):130-7.

29. Taylor BE, McClave SA, Martindale RG, Warren MM, Johnson DR, Braunschweig C, et al. Guidelines for the pro- 
vision and assessment of nutrition support therapy in the adult critically ill patient: society of critical care medicine (SCCM) and American society for parenteral and enteral nutrition (A.S.P.E.N.). Crit Care Med 2016; 44:390-438.

30. Thibault R, Picot D. Chyme reinfusion or enteroclysis in nutrition of patients with temporary double enterostomy or enterocutaneous fistula. Curr Opin Clin Nutr Metab Care 2016; 19(5):382-7.

31. Casaer MP, Mesotten D, Hermans G, Wouters PJ, Schetz M, Meyfroidt G, et al. Early versus late parenteral nutrition in critically ill adults. N Engl J Med 2011; 365:506e17.

32. Heidegger CP, Berger MM, Graf S, Zingg W, Darmon P, Costanza MC, et al. Optimisation of energy provision with supplemental parenteral nutrition in critically ill patients: a randomised controlled clinical trial. Lancet 2013; 381:385e93.

33. Doig GS, Simpson F, Sweetman EA, Finfer SR, Cooper DJ, Heighes PT, et al; Early PN Investigators of the ANZICS Clinical Trials Group. Early parenteral nutrition in critically ill patients with short-term relative contraindications to early enteral nutrition: a randomized controlled trial. JAMA 2013; 309:2130-8.

34. Allingstrup MJ, Kondrup J, Wiis J, Claudius C, Pedersen UG, Hein-Rasmussen R, et al. Early goal-directed nutrition versus standard of care in adult intensive care patients: the single-centre, randomised, outcome assessor-blinded EAT-ICU trial. Intensive Care Med 2017; 43:1637-47.

35. Ashkenazi I, Turégano-Fuentes F, Olsha O, Alfici R. Treatment options in gastrointestinal cutaneous fistulas. Surg J (N Y) 2017; 3(1):e25-e31.

36. Datta V, Engledow A, Chan S, Forbes A, Cohen CR, Windsor A. The management of enterocutaneous fistula in a regional unit in the United Kingdom: a prospective study. Dis Colon Rectum 2010; 53(2):192-9.
37. Goulet O, Dabbas-Tyan M, Talbotec C, Kapel N, Rosilio M, Souberbielle JC, et al. Effect of recombinant human growth hormone on intestinal absorption and body composition in children with short bowel syndrome. JPEN J Parenter Enteral Nutr 2010; 34(5):513-20.

38. Naberhuis JK, Tappenden KA. Teduglutide for safe reduction of parenteral nutrient and/or fluid requirements in adults: a systematic review. J Parenter Enter Nutr 2016; 40(8): 1096-05.

39. Jeppesen PB, Gilroy R, Pertkiewicz M, Allard JP, Messing B, O'Keefe SJ. Randomised placebo-controlled trial of teduglutide in reducing parenteral nutrition and/or intravenous fluid requirements in patients with short bowel syndrome. Gut 2011; 60(7):902-14.

40. Schwartz LK, O'Keefe SJ, Fujioka K, Gabe SM, Lamprecht G, Pape UF, et al. Long-term teduglutide for the treatment of patients with intestinal failure associated with short bowel syndrome. Clin Transl Gastroenterol 2016; 7(2):e142.

41. Martínez MI, Rumbo C, García D, Trentadue J, Gondolesi G, Fernández A. Experiencia con el uso de teduglutide en pacientes pediátricos con fallo intestinal en un centro de Argentina. Acta Gastroenterol Latinoam 2020; 50(3):257-63.

42. Kumar P. Classification of negative pressure wound therapy. Plast Aesthet Res 2019; 6:32.

43. Bobkiewicz A, Walczak D, Smolinski S, Kasprzyk T, Studniarek A, Borejsza-Wysocki M, et al. Management of enteroatmospheric fistula with negative pressure wound therapy in open abdomen treatment: a multicentre observational study. Int Wound J 2017; 14(1):255-64.

44. Haack CI, Galloway JR, Srinivasan J. Enterocutaneous fistulas: a look at causes and management. Appl Phys B 2014; 2(10):1-10.

\section{Contribución de autores}

Los autores participaron en igual medida en las distintas etapas elaboración del artículo

Aldo Miguel Agüero Milanés: https://orcid.org/0000-0002-4433-7786

Kenia Zusel Infante Rondón: https://orcid.org/0000-0002-3839-9972 